\title{
Backstepping Controller Design using a High Gain Observer for Induction Motor
}

\author{
Haj Brahim Imen \\ University of Sfax, \\ Automatic Control Unit, \\ National Engineering School \\ of Sfax, BP. 1173, 3038 Sfax, \\ Tunisia
}

\author{
Soufien Hajji \\ University of Sfax, \\ Automatic Control Unit, \\ National Engineering School \\ of Sfax, BP. 1173, 3038 Sfax, \\ Tunisia
}

\author{
Abdessattar Chaari \\ University of Sfax, \\ Automatic Control Unit, \\ Department of Electrical \\ Engineering, \\ National Engineering School \\ of Sfax, BP. 1173, 3038 Sfax, \\ Tunisia
}

\begin{abstract}
In this paper, a backstepping controller of Induction Motor (IM) is proposed using the fifth order model in fixed two frame reference axis with rotor flux and stator currents as state variables. The approach of backstepping requires, generally, that the nonlinear system is in strict feed-back loop. To implement this strategy over the IM, some transformations on the model $(\alpha, \beta)$ of the machine have been carried out without recourse to the oriented flux hypothesis which allows a triangular state representation. The overall system stability is proved by Lyapunov theory. Indeed the controller relationship depends on the unmeasured states of the IM, and a nonlinear observer to high gain is used in order to reconstruct the motor speed, the rotor flux and the load torque. Simulation results are provided to illustrate the effectiveness of the proposed approach and the robustness to uncertainties, such as rotor resistance variations.
\end{abstract}

\section{General Terms}

Backstepping controller design based high gain observer, Stability using Lyapunov theory, robustness of the backstepping controller to uncertainties in IM.

\section{Keywords}

Backstepping control, induction motor, high gain observer.

\section{INTRODUCTION}

In recent years, Backstepping approach became one of the most popular design methods for large scale nonlinear systems. The idea of backstepping design is to select recursively some appropriate functions of state variables as pseudo-control inputs for lower dimension subsystems of the overall system. Each backstepping stage results in a new pseudo-control design, expressed in terms of the pseudo- control designs from preceding design stages. When the procedure terminates, a feedback design for the true control input is the result which achieves the original design objective by virtue of final Lyapunov function, which is formed by summing up the Lyapunov functions associated with each individual design stage [1]. This control scheme can successfully guarantee the global asymptotic stability [2].

Applied to the control of IM, the strategy of backstepping can be used in two different ways. The first method utilizes the entire IM model without any simplifying assumption, which leads to a tedious analysis to construct a regression matrix [3]. These problems are taken care of by the introduction of the neuronal networks techniques to design the fictitious controller [4-5]. The second method works in combination with field oriented control FOC. Many versions of backstepping control have been developed. In [6-7-8], the PI controllers used in conventional FOC for speed and current regulation are replaced by backstepping controllers. In [9-10-11], the authors have extended the method to the adaptive neuronal network control systems in order to compensate the parameters variations and reject the external load torque disturbance.

One may note that the FOC methods represent a type of partial feedback linearization control technique in which the zero dynamic stability cannot be proved. As a result, it is not guaranteed that the system model is robust to parameters variation [4].

The interest of the backstepping controller, which is adopted in this paper, can be summarized in the fact that we have carried out some transformations on the model $(\alpha, \beta)$ of the machine allowing the state representation for a strict feed-back form. Based on the algorithm presented in [12], which requires that the nonlinear system must be in a triangular form, a novel approach of backstepping design is presented to control the speed and the rotor flux.

This paper is organized as follows: The detailed induction motor is presented in section 2. The nonlinear Backstepping control is summarized in section 3. In section 4, a high gain observer is given in order to estimate the unmeasured states. The simulation results, for performances evaluation, are illustrated and discussed in section 5 .

\section{INDUCTION MOTOR MODEL}

The fifth order IM model, in two fixed axis reference frame with rotor fluxes and stator currents as state variables [14], is given as:

$$
\left\{\begin{array}{l}
\dot{i}_{s}=-\gamma i_{s}+K\left(\frac{1}{T_{r}} I_{2}-n_{p} \Omega J_{2}\right) \psi_{r}+\frac{1}{\sigma L_{s}} u_{s} \\
\dot{\psi}_{r}=\frac{M}{T_{r}} i_{s}-\left(\frac{1}{T_{r}} I_{2}-n_{p} \Omega J_{2}\right) \psi_{r} \\
\dot{\Omega}=\frac{n_{p} M}{J L_{r}} i_{s}^{T} J_{2} \psi_{r}-\frac{1}{J} T_{l}
\end{array}\right.
$$


where $i_{s}=\left[\begin{array}{ll}i_{s \alpha} & i_{s \beta}\end{array}\right]^{T}, \psi_{r}=\left[\begin{array}{ll}\varphi_{r \alpha} & \varphi_{r \beta}\end{array}\right]^{T}, u_{s}=\left[\begin{array}{ll}u_{s \alpha} & u_{s \beta}\end{array}\right]^{T}$ are, respectively, the stator current, the rotor fluxes and the stator voltages; $\Omega$ and $T$ respectively denote the motor speed and the load torque; $f(\Omega)=\frac{1}{T_{r}} I_{2}-n_{p} \Omega J_{2}, I_{2}$ is the $2 \times 2$ matrix identity and $J_{2}=\left(\begin{array}{cc}0 & -1 \\ 1 & 0\end{array}\right) ; n_{p}$ is the number of pole pairs; $J$ is the motor moment of inertia. The parameters $T_{r}, \sigma, K$ and $\gamma$ are defined as:

$T_{r}=\frac{L_{r}}{R_{r}}, \sigma=1-\frac{M^{2}}{L_{s} L_{r}}, K=\frac{M}{\sigma L_{s} L_{r}}, \gamma=\frac{R_{s}}{\sigma L_{s}}+\frac{M^{2}}{\sigma L_{s} L_{r}^{2}}$

$L_{s}, L_{r}$ are per-phase stator and rotor inductances. $M$ is the mutual inductance. $R_{s}, R_{r}$ are stator and rotor resistances.

\section{BACKSTEPPING CONTROL}

The control objective consists in regulating the square of the fluxes vector norm at a desired constant value and forced the speed to follow a reference profile. Let us denote by $z^{1}=\left(\begin{array}{l}z_{1}^{1} \\ z_{2}^{1}\end{array}\right)=\left(\begin{array}{l}\Omega \\ \left\|\psi_{r}\right\|^{2}\end{array}\right)=\left(\begin{array}{l}\Omega \\ \varphi_{r \alpha}^{2}+\varphi_{r \beta}^{2}\end{array}\right)$ the vector variable to be controlled and let $z_{d}^{1}=\left(\begin{array}{c}z_{d 1}^{1} \\ z_{d 2}^{1}\end{array}\right)$ be the corresponding desired trajectory. With these notations, the control input should be defined to achieve asymptotically the following equation:

$$
\lim _{t \rightarrow \infty}\left(z_{1}^{1}(t)-z_{d 1}^{1}(t)\right)=\lim _{t \rightarrow \infty}\left(z_{2}^{1}(t)-z_{d 2}^{1}(t)\right)=0
$$

\subsection{Preliminary stage}

The backstepping approach provides a recursive method for stabilizing the origin of a system in strict-feedback form. In order to implement this strategy over the IM, we must go through a preliminary step in which we will make some transformations that bring the model of the IM in strict feedback form [13].

The new introduced coordinates are expressed by:

$$
\left\{\begin{array}{l}
z_{1}^{1}=\Omega \\
z_{2}^{1}=\left\|\psi_{r}\right\|^{2}=\varphi_{r \alpha}^{2}+\varphi_{r \beta}^{2}=\psi_{r}^{T} \psi_{r} \\
z_{1}^{2}=\frac{n_{p} M}{L_{r}} i_{s}^{T} J_{2} \psi_{r} \\
z_{2}^{2}=i_{s}^{T} \psi_{r} \\
\xi=\arctan \left(\frac{\varphi_{r \beta}}{\varphi_{r \alpha}}\right)
\end{array}\right.
$$

In a condensed form, system (1) can be written as:

$$
\left\{\begin{array}{l}
\dot{z}^{1}=A_{1} z^{2}+\Phi^{1}\left(C_{r}(t), z^{1}\right) \\
\dot{z}^{2}=b(\xi, z) u_{s}+g(z) \\
\dot{\xi}=\frac{L_{r}}{n_{p} T_{r}} \frac{z_{1}^{2}}{z_{2}^{1}}+n_{p} z_{1}^{1}
\end{array}\right.
$$

where:

$$
\begin{aligned}
& A_{1}=\left(\begin{array}{cc}
\frac{1}{J} & 0 \\
0 & \frac{2 M}{T_{r}}
\end{array}\right), \Phi^{1}\left(C_{r}(t), z^{1}\right)=\left(\begin{array}{c}
-\frac{1}{J} T_{l} \\
-\frac{2}{T_{r}} z_{1}^{2}
\end{array}\right) \\
& b(\xi, z)=\frac{1}{\sigma L_{s}} \sqrt{z_{2}^{1}}\left(\begin{array}{cc}
-\frac{n_{p} M}{L_{r}} \sin (\xi) & \frac{n_{p} M}{L_{r}} \cos (\xi) \\
\cos (\xi) & \sin (\xi)
\end{array}\right) \\
& g(z)=\left(\begin{array}{l}
g_{1}(z) \\
g_{2}(z)
\end{array}\right) \\
& g_{1}(z)=-n_{p}^{2} \frac{K M}{L_{r}} z_{1}^{1} z_{2}^{1}-\left(\gamma+\frac{1}{T_{r}}\right) z_{1}^{2}-n_{p}^{2} \frac{M}{L_{r}} z_{1}^{1} z_{2}^{2} \\
& g_{2}(z)=\frac{K}{T_{r}} z_{2}^{1}+\frac{L_{r}}{M} z_{1}^{1} z_{1}^{2}-\left(\gamma+\frac{1}{T_{r}}\right) z_{2}^{2} \\
& +\frac{M}{T_{r}} \frac{1}{z_{2}^{1}}\left(\left(z_{2}^{2}\right)^{2}+\left(\frac{L_{r}}{n_{p} M} z_{1}^{2}\right)^{2}\right)
\end{aligned}
$$

Let $z_{d}=\left(\begin{array}{c}z_{d}^{1} \\ z_{d}^{2}\end{array}\right)$ with $z_{d}^{2}=\left(\begin{array}{c}z_{d 1}^{2} \\ z_{d 2}^{2}\end{array}\right)$ be the trajectory of $z$ corresponding to the reference trajectory $z_{d 1}^{1}(t)$ and let $u_{d}$ be the associated input i.e. the input which brings $z(t)$ to the desired trajectory $z_{d}(t)$. According to system (3), $z_{d}^{2}$ and $u_{d}$ can be computed as:

$$
\left\{\begin{array}{l}
z_{d}^{2}=A_{1}^{-1}\left(\dot{z}_{d}^{1}-\Phi^{1}\left(T_{l}(t), z_{d}^{1}\right)\right) \\
b\left(\xi, z_{d}\right) u_{d}=\dot{z}_{d}^{2}-g\left(z_{d}\right)
\end{array}\right.
$$

This gives $z_{d}^{2}=\left(\begin{array}{l}J \dot{z}_{d 1}^{1}+C_{r} \\ \frac{T_{r}}{2 M} \dot{z}_{d 2}^{1}+\frac{1}{M} z_{d 2}^{1}\end{array}\right), \dot{z}_{d}^{2}=\left(\begin{array}{l}J \ddot{z}_{d 1}^{1}+\dot{C}_{r} \\ \frac{T_{r}}{2 M} \ddot{z}_{d 2}^{1}+\frac{1}{M} \dot{z}_{d 2}^{1}\end{array}\right)$

We define the following variables of errors:

$$
e^{1}=\left(\begin{array}{c}
e_{1}^{1} \\
e_{2}^{1}
\end{array}\right)=\left(\begin{array}{c}
z_{1}^{1}-z_{d 1}^{1} \\
z_{2}^{1}-z_{d 2}^{1}
\end{array}\right) ; e^{2}=\left(\begin{array}{c}
e_{1}^{2} \\
e_{2}^{2}
\end{array}\right)=\left(\begin{array}{c}
z_{1}^{2}-z_{d 1}^{2} \\
z_{2}^{2}-z_{d 2}^{2}
\end{array}\right)
$$

Based on (3), we can easily determine the errors dynamic: 


$$
\left\{\begin{array}{l}
\dot{e}^{0}=e^{1} \\
\dot{e}^{1}=A_{1} e^{2}+\Phi^{1}\left(C_{r}(t), z^{1}\right)+A_{1} z_{d}^{2}-\dot{z}_{d}^{1} \\
\dot{e}^{2}=b(\xi, z) u_{s}+g(z)-\dot{z}_{d}^{2}
\end{array}\right.
$$

After some mathematical operations, the IM model in errors space can be simplified as:

$$
\left\{\begin{array}{l}
\dot{e}^{0}=e^{1} \\
\dot{e}^{1}=A_{1} e^{2}+A_{2} e^{1} \\
\dot{e}^{2}=b(\xi, z) u_{s}+g(z)-\dot{z}_{d}^{2}
\end{array}\right.
$$

with $A_{2}=\left(\begin{array}{cc}0 & 0 \\ 0 & -\frac{2}{T_{r}}\end{array}\right)$.

\subsection{Control design}

Consider the following third order strict-feedback system.

$$
\left\{\begin{array}{l}
\dot{e}^{0}=e^{1} \\
\dot{e}^{1}=A_{1} e^{2}+A_{2} e^{1} \\
\dot{e}^{2}=b(\xi, z) u_{s}+g(z)-\dot{z}_{d}^{2}
\end{array}\right.
$$

The controller design procedure for induction motor can be given as follows.

Step1: Start with the first equation of (9), we define a new coordinate $y_{0}=e^{0}$ and derive its dynamic:

$$
\dot{y}_{0}=e^{1}
$$

We view $e^{1}$ as a control variable and define a virtual control law for equation (9), denote $\alpha_{0}$, and let $y_{1}$ be an error variable representing the difference between the actual and virtual controls:

$$
y_{1}=e^{1}-\alpha_{0}
$$

Our objective, in this step, is to design the stabilizing function $\alpha_{0}$ which makes $y_{0} \rightarrow 0$. The first Lyapunov candidate function $V_{0}$ is chosen as:

$$
V_{0}=\frac{1}{2} y_{0}^{T} y_{0}
$$

Its time derivative is given by:

$$
\dot{V}_{0}=y_{0}^{T} \dot{y}_{0}=y_{0}^{T} \alpha_{0}+y_{0}^{T} y_{1}
$$

To make the first order system stabilizable, it is necessary that the Lyapunov function derivative $\dot{V}_{0}$ is negative, which will allow the following choice.

$$
\alpha_{0}=-c_{0} y_{0}
$$

Then the time derivative of $V_{0}$ becomes:

$$
\dot{V}_{0}=-c_{0} y_{0}^{T} y_{0}+y_{0}^{T} y_{1}
$$

where $c_{0}$ is a positive constant.

In order to obtain $\dot{V}_{0}<0$ and guaranteed that $y_{0}$ converge to zero asymptotically, the residual term $y_{0}^{T} y_{1}$ will be compensated in the second step.

Step2: We derive the error dynamics for $y_{1}=e^{1}-\alpha_{0}$

$$
\begin{aligned}
\dot{y}_{1} & =\dot{e}^{1}+c_{0} e^{1} \\
& =A_{1} e^{2}+A_{2} e^{1}+c_{0} e^{1}
\end{aligned}
$$

Define a second virtual control law $\alpha_{1}$ and let $y_{2}$ be an error variable representing the difference between the actual and virtual controls.

$$
y_{2}=e^{2}-\alpha_{1}
$$

Then the error equation (15) can be expressed as:

$$
\dot{y}_{1}=A_{1} y_{2}+A_{1} \alpha_{1}+A_{2} e^{1}+c_{0} e^{1}
$$

The control objective is to make $y_{1}$ converge to zero. We extend the initial Lyapunov function to reflect the presence of the new state variable $y_{1}$ as:

$$
V_{1}=V_{0}+\frac{1}{2} y_{1}^{T} y_{1}
$$

Then, its derivative is:

$$
\begin{aligned}
\dot{V}_{1} & =\dot{V}_{0}+y_{1}^{T} \dot{y}_{1} \\
& =-c_{0} y_{0}^{T} y_{0}+y_{0}^{T} y_{1}+y_{1}^{T}\left(A_{1} y_{2}+A_{1} \alpha_{1}+A_{2} e^{1}+c_{0} e^{1}\right) \\
& =-c_{0} y_{0}^{T} y_{0}+y_{1}^{T}\left(y_{0}+A_{1} \alpha_{1}+A_{2} e^{1}+c_{0} e^{1}\right)+y_{1}^{T} A_{1} y_{2}
\end{aligned}
$$

We can now select an appropriate virtual control $\alpha_{1}$ to cancel some terms related to $y_{0}$ and $e^{1}$ :

$$
\alpha_{1}=-A_{1}^{-1}\left(c_{1} y_{1}+y_{0}+\left(A_{2}+c_{0} I_{2}\right) e^{1}\right)
$$

where $c_{1}$ is a positive constant. So the time derivative of $V_{1}$ becomes:

$$
\dot{V}_{1}=-c_{0} y_{0}^{T} y_{0}-c_{1} y_{1}^{T} y_{1}+y_{1}^{T} A_{1} y_{2}
$$

Clearly, if $y_{2}=0$ we obtain $\dot{V}_{1}=-c_{0} y_{0}^{T} y_{0}-c_{1} y_{1}^{T} y_{1}$, and thus both $y_{0}$ and $y_{1}$ are guaranteed to converge to zero asymptotically. 
Step3: We proceed in the same way with the last equation in (8) and we start by computing the error dynamics for $y_{2}=e^{2}-\alpha_{1}$ proposed in step2:

$$
\begin{aligned}
\dot{y}_{2} & =\dot{e}^{2}-\dot{\alpha}_{1} \\
& =b(\xi, z) u_{s}+g(z)-\dot{z}_{d}^{2}-\dot{\alpha}_{1}
\end{aligned}
$$

We note the appearance of the real control $u_{s}$. In this step, our objective is to design the actual control input such that $y_{2}$ converge to zero. So, we need so to select a new Lyapunov function to design the final control.

$$
V_{2}=V_{1}+\frac{1}{2} y_{2}^{T} y_{2}
$$

Its derivative is given by:

$$
\begin{aligned}
& \dot{V}_{2}=\dot{V}_{1}+y_{2}^{T} \dot{y}_{2} \\
& =-c_{0} y_{0}^{T} y_{0}-c_{1} y_{1}^{T} y_{1} \\
& +y_{2}^{T}\left(b(\xi, z) u_{s}+A_{1} y_{1}+g(z)-\dot{z}_{d}^{2}-\dot{\alpha}_{1}\right)
\end{aligned}
$$

To stabilize the global system (9), the real control input is selected to remove the residual term and make $\dot{V}_{2}<0$.

$$
u_{s}=(b(\xi, z))^{-1}\left(-c_{2} y_{2}-A_{1} y_{1}+\dot{z}_{d}^{2}+\dot{\alpha}_{1}-g(z)\right)
$$

Where $c_{2}$ is a positive constant. The derivative of the candidate Lyapunov function becomes:

$$
\dot{V}_{2}=-c_{0} y_{0}^{T} y_{0}-c_{1} y_{1}^{T} y_{1}-c_{2} y_{2}^{T} y_{2}
$$

Therefore the asymptotic convergence to zero of $y_{0}, y_{1}$ and $y_{2}$ is guaranteed. Since $y_{0}=e^{0}, e^{0}$ is also bounded, and $\lim e^{0}=0$. The boundedness of $e^{1}$ follows from the boundedness of $\alpha_{0}$ in (13) and the fact that $e^{1}=y_{1}+\alpha_{0}$ so, $\lim e^{1}=0$. Similarly, the boundedness of $e^{2}$ then follows from boundedness of $\alpha_{1}$ in (20) and the fact that $e^{2}=y_{2}+\alpha_{1}$. So, we have $\lim _{t \rightarrow \infty} e^{2}=0$.

From the above we can obtain the control law as:

$$
u_{s}=(b(\xi, z))^{-1}\left(-c_{2} y_{2}-A_{1} y_{1}+\dot{z}_{d}^{2}+\dot{\alpha}_{1}-g(z)\right)
$$

Now, it suffices to replace, in the final control law, input $z$ and $e$ by their respective expressions in the original coordinates, namely (2) and (5).

\section{HIGH GAIN OBSERVER}

The control law depends on unmeasured states; a nonlinear observer shall be synthesized in order to achieve the estimation objective. In fact the proposed observer [14] consists of two cascade observers. The first observer provides the estimation of rotor fluxes using measurement of stators currents and motor speed. The second cascade observer provides the estimation of the load torque and its derivative.

The measured output vector is $y=\left(\begin{array}{l}i \\ \Omega \\ \Omega\end{array}\right)$. A high gain observer can be synthesized in order to provide the estimation of $\psi_{r}$, and its equations are:

$$
\left\{\begin{array}{l}
\dot{\hat{i}}_{s}=K f(\Omega) \hat{\psi}_{r}-\gamma \hat{i}_{s}+\frac{1}{\sigma L_{s}} u_{s}-2 \theta_{1}\left(\hat{i}_{s}-i_{s}\right) \\
\dot{\hat{\psi}}_{r}=\frac{M}{T_{r}} \hat{i}_{s}-f(\Omega) \hat{\psi}_{r}-\frac{\theta_{1}^{2}}{K} f^{-1}(\Omega)\left(\hat{i}_{s}-i_{s}\right)
\end{array}\right.
$$

where $\hat{i}_{s}$ and $\hat{\psi}_{r}$ are the respective estimates of $i_{s}, \psi_{r}$, and $\theta_{1}$ is the parameter design.

The flux estimates are then used together with the motor speed measurements in order to estimate the load torque $T_{L}$ and its time derivative $T_{L p}$ using the following nonlinear observer:

$$
\left\{\begin{array}{l}
\dot{\hat{\Omega}}=\frac{n_{p} M}{J_{L_{r}}} \hat{i}_{s}^{T} J_{2} \hat{\psi}_{r}-\frac{1}{J} \hat{T}_{L}-3 \theta_{2}(\hat{\Omega}-\Omega) \\
\dot{\hat{T}}_{L}=\hat{T}_{L p}+3 \theta_{2}^{2} J(\hat{\Omega}-\Omega) \\
\dot{\hat{T}}_{L p}=\theta_{2}^{3} J(\hat{\Omega}-\Omega)
\end{array}\right.
$$

where $\hat{T}_{L}$ and $\hat{T}_{L p}$ are the respective estimates of $T_{L}, T_{L p}$, and $\theta_{2}$ is the parameter design.

Notice that the estimation error converges exponentially to zero for observer (30). The main characteristic of observer (31) lies in the fact that when the time derivative of the load torque is constant, the estimation error converges to zero exponentially. When this time derivative is not constant but remains bounded by a constant, the estimation error can be made as small as desired by choosing $\theta_{2}$ high enough [13].

\section{SIMULATION RESULTS}

The proposed control algorithm has been simulated for a $1 \mathrm{~kW}$ induction motor in order to prove the rightness and effectiveness of the designed controller.

The gains $\left[\begin{array}{lllll}\theta_{1} & \theta_{2} & c_{0} & c_{1} & c_{2}\end{array}\right]$ are chosen as follows: $\left[\begin{array}{lllll}2500 & 500 & 5000 & 500 & 5000\end{array}\right]$ to satisfy convergence conditions. The simulation parameters of IM are givens as:

$n_{p}=2 ; J=0.015 \mathrm{Kgm}^{2} ; M=0.29 \mathrm{H}$

$R_{s}=10.6 \Omega ; R_{s}=2.88 \Omega ; L_{s}=L_{r}=0.3 H$

Simulation results, with nominal value of rotor resistance, are reported in figure 1 to 3 . The obtained result shows that the tracking performance of speed and flux are very satisfactory for various forms of reference speed (low speed, high-speed and 
reverse speed). Notice that the tracking error related to the motor speed tends rapidly to zero after each sudden change of load torque. The performances of the observer are illustrated in figure 2 and 3 where the estimation error of $\left\|\psi_{r}\right\|^{2}$ and the load torque are given.
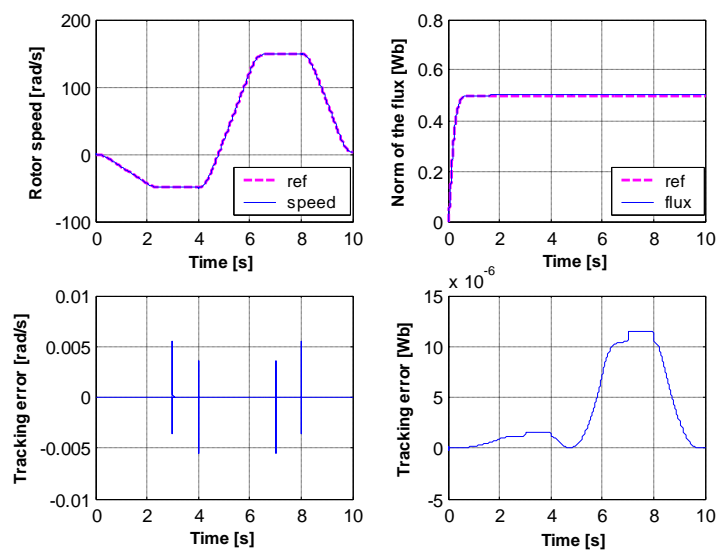

Fig1: Time evolution of the rotor speed, the fluxes norm and their tracking error
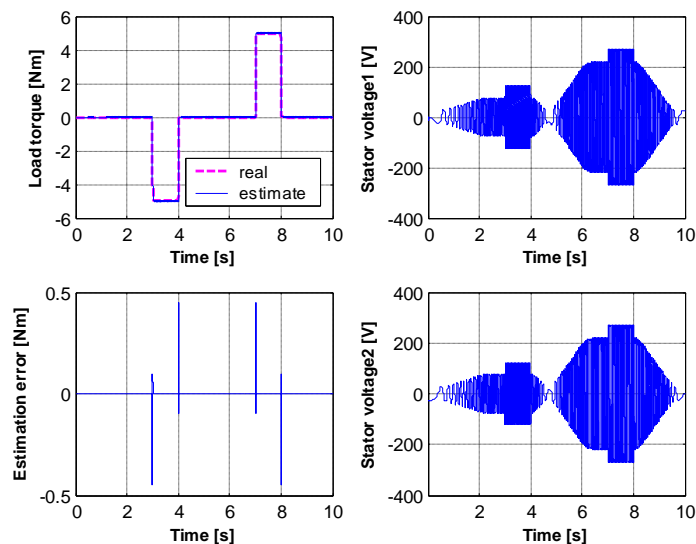

Fig2: Time evolution of the load torque and the voltage input

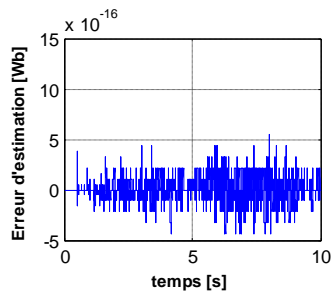

Fig3: Estimation error of $\left\|\psi_{r}\right\|^{2}$

To test the effect of rotor resistance on the controller, its variation (figure 6-a) is injected into the model of IM while its nominal value is retained in the expression of the controller and the observer. The obtained results (figures 4, 5, 6-b) are quite similar to those obtained in the first case. This proves the robustness and effectiveness of the proposed control law.
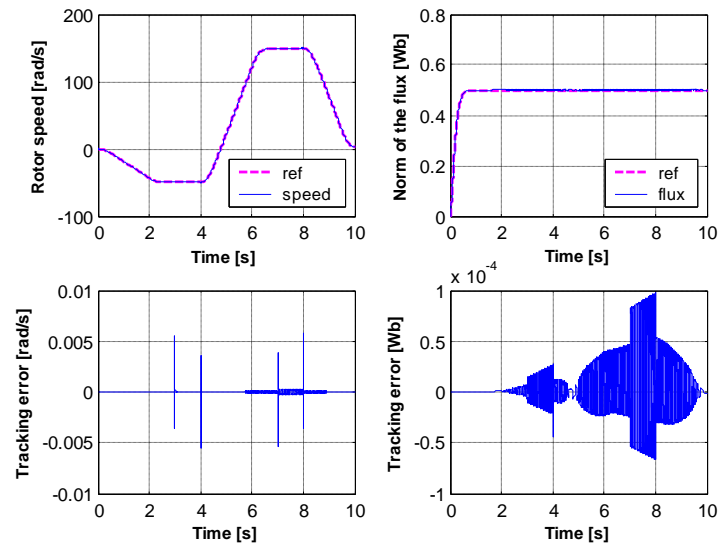

Fig4: Time evolution of the rotor speed, the fluxes norm and their tracking error with rotor resistance variation.
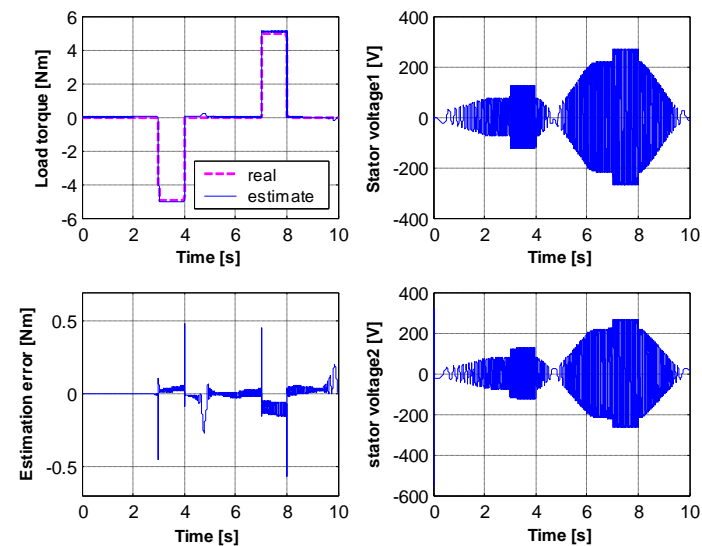

Fig5: Time evolution of the load torque and the voltage input with rotor resistance variation.
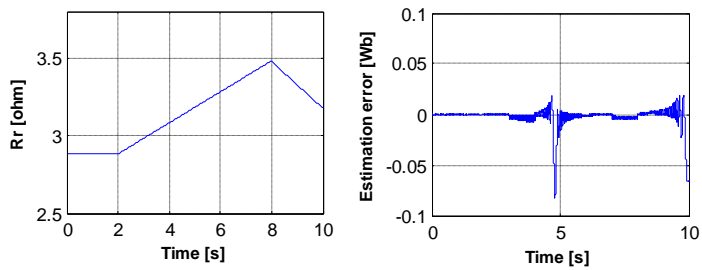

Fig6: Variation of rotor resistance (a), Estimation error of $\left\|\psi_{r}\right\|^{2}$ with rotor resistance variation (b)

\section{CONCLUSION}

In this paper, a backstepping controller has been proposed for IM rotor flux and speed tracking control. The nonlinear controller is designed based on the fifth order IM model in two fixed axis reference frame. The validity of this design is demonstrated through computer simulations. The obtained results confirm also the robustness of the proposed control law against the variation of rotor resistance. 


\section{APPENDIX}

The expression of the final control input:

$$
\begin{aligned}
& u_{s}=(b(\xi, z))^{-1}(\underbrace{-c_{2} y_{2}-A_{1} y_{1}+\dot{z}_{d}^{2}+\dot{\alpha}_{1}}_{h}-g(z)) \\
& (b(\xi, z))^{-1}=\frac{\sigma L_{s} L_{r}}{n_{p} M z_{2}^{1}}\left(\begin{array}{ll}
-\varphi_{r \beta} & \frac{n_{p} M}{L_{r}} \varphi_{r \alpha} \\
\varphi_{r \alpha} & \frac{n_{p} M}{L_{r}} \varphi_{r \beta}
\end{array}\right) \\
& g(z)=\left(\begin{array}{l}
g_{1}(z) \\
g_{2}(z)
\end{array}\right) \\
& g_{1}(z)=-n_{p}^{2} \frac{K M}{L_{r}} z_{1}^{1} z_{2}^{1}-\left(\gamma+\frac{1}{T_{r}}\right) z_{1}^{2}-n_{p}^{2} \frac{M}{L_{r}} z_{1}^{1} z_{2}^{2} \\
& g_{2}(z)=\frac{K}{T_{r}} z_{2}^{1}+\frac{L_{r}}{M} z_{1}^{1} z_{1}^{2}-\left(\gamma+\frac{1}{T_{r}}\right) z_{2}^{2} \\
& +\frac{M}{T_{r}} \frac{1}{z_{2}^{1}}\left(\left(z_{2}^{2}\right)^{2}+\left(\frac{L_{r}}{n_{p} M} z_{1}^{2}\right)^{2}\right) \\
& h=-\left(\begin{array}{l}
k_{01} e_{1}^{0}+k_{11} e_{1}^{1}+k_{21} e_{1}^{2}-\dot{C}_{r}-J \ddot{z}_{d 1}^{1} \\
k_{02} e_{2}^{0}+k_{12} e_{2}^{1}+k_{22} e_{2}^{2}-\frac{T_{r}}{2 M} \ddot{z}_{d 2}^{1}-\frac{1}{M} \dot{z}_{d 2}^{1}
\end{array}\right) \\
& k_{01}=\left(J c_{1} c_{0} c_{2}+J c_{2}+c_{0} \frac{1}{J}\right) \\
& k_{02}=\left(\frac{T_{r}}{2 M} c_{1} c_{0} c_{2}+\frac{T_{r}}{2 M} c_{2}+c_{0} \frac{2 M}{T_{r}}\right) \\
& k_{11}=\left(J c_{1} c_{2}+J c_{0} c_{2}+\frac{1}{J}+J c_{1} c_{0}+J\right) \\
& k_{12}=\frac{T_{r}}{2 M}\left(c_{1} c_{2}+c_{0} c_{2}+c_{1} c_{0}+1\right)+\frac{2 M}{T_{r}} \\
& +\frac{2}{M T_{r}}-\frac{1}{M}\left(c_{1}+c_{0}+c_{2}\right) \\
& k_{21}=\left(c_{2}+c_{1}+c_{0}\right) ; k_{22}=\left(c_{2}+c_{1}+c_{0}-\frac{2}{T_{r}}\right)
\end{aligned}
$$

\section{REFERENCES}

[1] N. Ezziani, A. Hussain, N. Essounbouli, A. Hamzaoui. Backstepping Adaptive Type-2 Fuzzy Controller for Induction Machine. ISIE 2008.

[2] R. Trabelsi, T. Gallah, A. Khedher, M. F. Mimouni and F. M'Sahli. Real-time implementation of Backstepping Control for an Induction Motor drive using dSpace DS-
1104 control board. 10th International Conference on Sciences and Techniques of Automatic control \& computer engineering. STA09. TUNISIA.

[3] F. Mehazzem, A. Reama, Y. Hamam and H.Benalla. Real time Implementation of Backsteing Controller in Indirect field oriented control of Induction motor drive. International Conference on Power Engineering, Energy and Electrical Drives, 2009. POWERENG '09.

[4] B J. Soltani, R. Yazdanpanah. Robust Backstepping Control of Induction Motor Drives using Artificial Neural Networks. Power Electronics and Motion Control Conference, 2006. IPEMC 2006. CES/IEEE 5th International.

[5] Z. C. Ji, Y. X. Shen. Backstepping Position Control for Induction Motor based on Neural Network. ICIEA 2006

[6] I. K. Bousserhane, A. Hazzab, M. Rahli, M. Kamli and B. Mazari. Direct Field-Oriented Control Design using Backstepping Technique for Induction Motor Speed Control. 14th Mediterranean Conference on Control and Automation MED '06. IEEE, 2006.

[7] S. Chaouch, L. Chrifi, A. Makouf, and M.S Nait. S. Backstepping control analysis of two different speed sensorless approaches for induction motor. 5th International Multi-Conference on Systems, Signals and Devices. IEEE SSD 2008.

[8] H. Tan, and J. Chang. Field Orientation and Adaptive Backstepping for Induction Motor Control. International Conference on Power Engineering, Energy and Electrical Drives, 2009. POWERENG '09.

[9] R. J.Wai, F. J. Lin and S. P. Hsu. Intelligent backstepping control for linear induction motor drive. IEE Proc.-Control Theory Appl., Vol. 148, No. 3, May 2001.

[10] N. Ezziani, N. Essounbouli, and A. Hamzaoui. Backstepping Fuzzy Adaptive Controller of Induction Machine. 6th Mediterranean Conference on Control and Automation Congress Centre, Ajaccio, France. June 25-27, 2008 .

[11] Y. H. Hwang, K. K. Park, H. W. Yang. Robust Adaptive Backstepping Control for Efficiency Optimization of Induction Motors with Uncertainties.

[12] J. Zhou, C. Wen. Adaptive. Backstepping control of uncertain systems, LNCIS 372, pp. 9-31. Springer 2008.

[13] W. Leonard. Control of electrical drives. Springer-Verlag, 2001. 3rd edition.

[14] J. Massieu, P. Dorlèans, S. Hajji, M. M'Saad and M. Farza. High-gain-based output feedback controllers for the induction motor. Fourth IEEE International MultiConference on Systems, Signals \& Devices (SSD), Tunisia, 2007. 